\title{
'Cool Little Kids' helps reduce later anxiety symptoms but not broader internalising problems
}

\author{
By Dr. Jessica Edwards
}

Children with a shy/inhibited temperament are at risk of developing internalising problems later in life. ${ }^{1}$ Unfortunately, the responses to such behaviours by some parents - such as overprotective or harsh parenting - can add to this risk. ' 'Cool Little Kids' is a group parenting intervention that comprises six, 90-minute manualised sessions delivered over 3 months that are led by clinical psychologists. ${ }^{3}$ These sessions provide information on child anxiety, anxiety management and parenting methods. The aim is to address the risks of internalising problems early in life in inhibited children.

Researchers in Australia now have new follow-up data for a novel community study of 'Cool Little Kids'. This randomised controlled trial initially involved 545 parents of temperamentally inhibited pre-schoolers (age 4 years) recruited from eight diverse areas across Melbourne, Australia. Parents of all children identified by universal pre-school screening for inhibition were invited to participate. There was an $86 \%$ retention rate at the 2 -year followup (age 6 years), resulting in data being available from 218 parents who had been allocated to the intervention arm and 245 allocated to the control arm (usual access to community support services). The data showed that at age 6 years, child anxiety symptoms were significantly reduced in the intervention versus the control arm. However, there was no significant impact on broader child internalising problems, anxiety disorders, parenting practices or parent distress.

The researchers noted that some families engaged less with the intervention than others. Families who had reduced attendance tended to include younger mothers, less educated or more culturally diverse fathers, and parents with lower household income. Families who less frequently practised skills after the programme included parents of girls, and those from more advantaged neighbourhoods. The researchers propose that a low level of engagement with the intervention could potentially impact on its effectiveness over the long term. Why particular family characteristics influence engagement in such parenting groups to prevent child internalising remains to be determined. For now, the researchers propose considering motivation techniques to engage these subgroups of families.

Referring to:

Bayer, J.K., Prendergast, L.A., Brown, A., Harris, L., Bretherton, L. Hiscock, H., Beatson, R., Mihalopoulous, C. \& Rapee, R.M. (2020), Cool Little Kids translational trial to prevent internalising: two-year outcomes and prediction of parent engagement. Child Adolesc. Ment. Health. doi: 10.1111/ camh.12420.

\section{References:}

${ }^{1}$ Claus, J.A., \& Blackford, J.U. (2012). Behavioural inhibition and risk for developing social anxiety disorder: A meta-analytic study. Journal of the American Academy of Child and Adolescent Psychiatry, 51, 1066-1075.

${ }^{2}$ Murray, L. et al. (2009), The development of anxiety disorders in childhood: An integrative review. Psychological Medicine, 39, 1413-1423.

${ }^{3}$ Rapee, R.M. (2013). The preventive effects of a brief, early intervention for preschool-aged children at risk for internalising: Follow-up into middle adolescence. Journal of Child Psychology and Psychiatry, 54, 780788.

\section{Glossary:}

Internalising problems: emotional symptoms which tend to be expressed internally, such as anxiety and low mood. 